\title{
La investigación del médico clínico en la Región Loreto
}

\author{
Research of clinician in the Peruvian Amazon region
}

\section{Sr. Editor:}

Con respecto a la carta al editor "Labor asistencial e investigación como pilares del quehacer diario del clínico" (1) publicada en la Revista Médica Herediana, consideramos oportunos los comentarios de los autores, pero debemos hacer algunas precisiones cuando se trabaja en un escenario diferente de la ciudad de Lima.

En Loreto, existen muchos profesionales médicos muy capacitados en el área clínica y de la salud pública, que a pesar de las limitaciones logísticas y de infraestructuras que puedan tener, realizan un gran trabajo. Pero, cuando nos referimos a investigación (clínica, operativa, epidemiológico, etc.) observamos que, son pocos los médicos clínicos de nuestra región que la realizan, y es probable que se deba a varios factores en su formación y al escaso tiempo que disponen, pero lo primero podría ser subsanable con el apoyo de investigadores médicos y no médicos. $\mathrm{Y}$ entonces, se hace muy importante, trabajar en redes, asociados a otros clínicos o profesionales que han demostrado mayor experiencia en el área de investigación (2).

Loreto, tiene un historial muy extenso con muchas instituciones de gran trayectoria en investigación como lo son la Universidad Peruana Cayetano Heredia, NAMRU-6, Instituto Nacional de Salud, PRISMA, y otros que cuentan con médicos investigadores de gran y moderada experticia en áreas clínica, epidemiológica, de laboratorio, con los cuales se podría establecer redes de investigación para el propósito de "investigar" para mejorar la salud. Los temas a investigar deberían estar encausados dentro de las prioridades de investigación, regionales o nacionales, para que puedan tener mayor posibilidad de aceptación en el logro del financiamiento y publicación $(3,4)$. Los convenios o la formación de círculos de investigación o centros de excelencia promovidos por CONCYTEC tienen esta finalidad (5).

El punto de partida inicial, debería ser la iniciativa de médicos locales para convocar la participación de otros investigadores de estas instituciones, de mayor trayectoria, en la realización elaboración conjunta de protocolos de investigación sobre temas de interés regional o local. La única condición, debiera ser el de obtener un beneficio mutuo, a través de la transferencia de conocimientos y/o de tecnología, y que finalmente, debería contribuir en la formación o consolidación de investigadores locales y su presencia de los mismos como autores, y coautores en protocolos de investigación y principalmente en publicaciones (2).

La investigación podría ser, inicialmente descriptiva, luego analítica, pero una vez que se tiene la intención de publicar datos, el siguiente paso es elaborar protocolos de investigación, y solicitar autorización de comités de ética, con la finalidad de proteger al sujeto de investigación y de poder garantizar la calidad de los datos al usar fichas ya validadas de recolección de datos. Para evaluar factores asociados, costos y percepciones, los estudios analíticos serán suficientes, sin embargo, para evaluar eficacia de los tratamientos farmacológicos, aspecto muy importante para los clínicos, se requiere de estudios experimentales, cuya factibilidad es, actualmente, muy difícil debido a las exigencias del reglamento nacional vigente (6). Y, consideramos que las investigaciones operativas son instrumentos importantes y necesarios que nos permiten comparar resultados e impacto de las medidas establecidas tanto en establecimientos de primer o tercer nivel, y son estas las que deberían fomentarse mediante fondos concursables, jornadas científicas o facilidades de la institución (horas laborales en investigación) para sus nuevos investigadores. Una política regional e 
institucional de reconocer esas horas brindadas a la investigación en temas relevantes para el mejoramiento del servicio, de los pacientes o del establecimiento, sería el mejor estimulo, pero a cambio, debe traducirse en datos confiables y publicables, lo que traducirá en prestigio personal e institucional.

Si bien es cierto, CONCYTEC está ofreciendo mayores oportunidades de fondos, la calidad de los proyectos y la confianza que generen los investigadores que participan, será clave para obtener financiamiento. Y, siendo nuestra región productora de petróleo, aún es incierto, como el Canon contribuye a la posibilidad de investigación operativa en salud que se necesita en nuestra región (7). Formemos redes y busquemos espacios para realizar investigación en salud.

\section{Nataly Atarama 1,2,a, Jury Puerta, ${ }^{1,2, a}$, Zuleyka Arica $^{1,2, a}$, Stalin Vilcarromero ${ }^{1,3, b, c}$}

\section{Descargo de responsabilidad:}

Las opiniones y afirmaciones contenidas aquí son propias de los autores y no deben interpretarse como posición oficial o que reflejan la opinión del Ministerio de Salud del Perú, del Departamento de la Marina, o del servicio naval de los Estados Unidos.

\section{Correspondencia:}

Stalin Vilcarromero

Av. La Marina c/Trujillo (Clínica Naval).

Iquitos-Loreto, Perú.

Correo electrónico: stalinvil@gmail.com

Teléfono: (51)-065601470 anexo 111 / Fax: (51)065601473

\section{REFERENCIAS BIBLIOGRÁFICAS}

1. León F, Osada J. Labor asistencial e investigación como pilares del quehacer diario del clínico. Rev Med Hered. 2014; 25:109-110.
2. Huamani C, Mayta-Tristan P. Producción científica peruana en medicina y redes de colaboración, análisis del Science Citation Index 2000-2009. Rev perú med exp salud publica. (Internet). 2010 (Citado el 21 de octubre del 2014); 27(3):315-325. Disponible en: http://www.scielo.org.pe/scielo.php?script=sci arttext\&pid=S1726-46342010000300003\&lng=es\& $\mathrm{nrm}=\mathrm{iso}$

3. Consejo Nacional de Ciencia, Tecnología e Innovación Tecnológica (CONCYTEC). Ministerio de Salud. Health priorities for Peru. Lima, Perú: CONCYTEC. (Citado el 21 de octubre de 2014). Disponible en: http://portal.concytec.gob.pe/images/stories/ images 2014/portal/areas-institucion/fondecyt/ideasaudaces/prioridades_de_salud_en_el_peru.pdf

4. Caballero P, Yagui $\bar{M}$, Espinoza $\bar{M}$, et al. Prioridades regionales y nacionales de investigación en salud, Perú 2010-2014: un proceso con enfoque participativo y descentralista. Rev perú med exp salud publica (Internet). 2010 (Citado el 21 de octubre del 2014); 27(3):398-411. Disponible en: http://www. scielo.org.pe/scielo.php?script=sci arttext\&pid=S1726-46342010000300013\&lng=es\& nrm $=$ iso

5. Consejo Nacional de Ciencia, Tecnología e Innovación Tecnológica. Círculos de Investigación. Lima, Perú: CONCYTEC; 2014. http://www.cienciactiva.gob.pe/ cientificos-inc.php

6. Instituto Nacional de Salud. Lima Reglamento de Ensayos Clínicos. Lima, Perú: Instituto Nacional de Salud, 2010. (Citado el 21 de octubre del 2014) Disponible en: http://www.ins.gob.pe/ repositorioaps/0/2/not/-1/Libro\%20Reglamento\%20 ensayos\%20clinicos.pdf

7. Romani F, Cabezas C, Espinoza M, et al. Strategy to strengthen health research capabilities in regional public universities: the role of the mining canon and the Instituto Nacional de Salud. Rev perú med exp salud publica (Internet). 2012 (Citado el 21 de octubre de 2014); 29(4): 549-554. Disponible en: http://www. scielo.org.pe/scielo.php?script=sci arttext\&pid=S1726-46342012000400021\&lng=es\& $\mathrm{nrm}=\mathrm{iso}$

Recibido: 20/10/2014

\footnotetext{
Sociedad Científica de Estudiantes de Medicina de la Universidad Nacional de la Amazonia Peruana. Iquitos, Perú. Facultad de Medicina, Universidad Nacional de la Amazonia Peruana. Iquitos, Perú

Naval Medical Research Unit N 6 (NAMRU-6). Iquitos, Perú.

Estudiante de Medicina; ${ }^{\mathrm{b}}$ Médico; ${ }^{\mathrm{c}}$ Magister en Salud Pública
} 